\title{
Avaliação dos Resultados do Tratamento Antiangiogénico na Neovascularização Coroideia Associada à Miopia Patológica
}

\author{
Evaluation of Antiangiogenic Treatment Results in Choroidal Neovascularization \\ Related to Pathological Myopia
}

\author{
Beatriz CARVALHO ${ }^{1}$, Paulo FREITAS-COSTA ${ }^{1}$, João PINHEIRO-COSTA ${ }^{1}$, Manuel FALCÃO ${ }^{1}$, Ângela CARNEIRO ${ }^{1}$, \\ Fernando FALCÃO-REIS ${ }^{1}$ \\ Acta Med Port 2014 Jan-Feb;27(1):49-58
}

\begin{abstract}
RESUMO
Introdução: A neovascularização coroideia associada à miopia patológica é uma das principais causas de perda de visão central e irreversível em indivíduos jovens. Os objetivos deste estudo são avaliar os resultados a longo prazo do tratamento antiangiogénico, com ranibizumab e/ou bevacizumab, na neovascularização coroideia associada à miopia patológica e caraterizar os fatores preditivos dos resultados funcionais e anatómicos obtidos.

Material e Métodos: Avaliámos os resultados de 84 olhos de 81 doentes com neovascularização coroideia miópica, dos quais 84 (100\%) completaram 12 meses de seguimento, 67 (79,8\%) 24 meses de seguimento, 54 (64,3\%) 36 meses de seguimento, 29 (34,5\%) 48 meses de seguimento e $15(16,7 \%) 60$ meses de seguimento. Procedemos à recolha de dados relativos à melhor acuidade visual corrigida em escala ETDRS, espessura foveal na tomografia de coerência óptica e caraterísticas da angiografia fluoresceínica, inicial e após tratamento.
\end{abstract}

Resultados: As melhorias na melhor acuidade visual corrigida e na espessura foveal foram significativas para todos os tempos de seguimento $(p<0,05)$. A média da melhor acuidade visual corrigida inicial era de $43,7 \pm 20,1$ letras e da espessura foveal inicial de $304,8 \pm 127,9 \mu \mathrm{m}$. As médias da melhor acuidade visual corrigida foram de 55,6 $\pm 18,5,52,1 \pm 22,3,52,1 \pm 22,6,50,3 \pm 23,8$ e 47,8 \pm 24,5 para os $12,24,36,48$ e 60 meses de tratamento, respetivamente. As médias das espessuras foveais foram de $209,7 \pm 86,2,190,6$ $\pm 76,1,174,7 \pm 60,6,189,8 \pm 96,7$ e 159,4 $\pm 73,3$ para os mesmos tempos de seguimento. Apenas a melhor acuidade visual corrigida inicial foi preditiva de melhores resultados na melhor acuidade visual corrigida final $(p<0,001)$.

Discussão/Conclusão: As injecções intravítreas de anti-VEGF em doentes com neovascularização coroideia miópica cursam com uma melhoria funcional e anatómica significativa e sustentada no tempo. Ensaios clínicos randomizados com follow-up mais extenso são necessários para comprovar a eficácia sustentada destes agentes.

Palavras-chave: Acuidade Visual; Anticorpos Monoclonais Humanizados; Injecções Intravítreas; Miopia Degenerativa; Neovascularização Coroideia; Bevacizumab; Ranibizumab.

\section{ABSTRACT}

Introduction: Choroidal neovascularization secondary to pathological myopia is one of the leading causes of irreversible central vision loss in younger patients. The purposes of our study is to evaluate the long-term results of antiangiogenic treatment, with ranibizumab and/or bevacizumab, in myopic choroidal neovascularization and define the predictive factors for visual and anatomic outcomes.

Material and Methods: In this study were included 84 eyes from 81 patients with myopic choroidal neovascularization. Eighty-four (100\%) eyes accomplish 12 months of follow-up, 67 (79.8\%) 24 months, 54 (64.3\%) 36 months, 29 (34.5\%) 48 months, and 15 (16.7\%) 60 months. We retrieved data related to best corrected visual acuity measured with ETDRS chart, foveal center thickness on optical coherence tomography and fluorescein angiographic findings, before and after treatment.

Results: The best corrected visual acuity and foveal center thickness improvements were statistically significant for all follow-up times $(p<0.05)$. Mean baseline best corrected visual acuity was $43.7 \pm 20.1$ letters and mean baseline foveal center thickness was $304.8 \pm$ $127.9 \mu \mathrm{m}$. Mean best corrected visual acuity was $55.6 \pm 18.5,52.1 \pm 22.3,52.1 \pm 22.6,50.3 \pm 23.8$ and $47.8 \pm 24.5$ for $12,24,36,48$ and 60 months of treatment, respectively. Mean foveal center thickness was $209.7 \pm 86.2,190.6 \pm 76.1,174.7 \pm 60.6,189.8 \pm 96.7$ and $159.4 \pm 73.3$ for the same follow-up times. Baseline best corrected visual acuity was the only predictive factor for better visual outcome $(p<0.001)$.

Discussion/Conclusion: Intravitreal anti-VEGF injections in patients with myopic choroidal neovascularization yielded a significant and sustained functional and anatomic improvement. Randomized long-term clinical trials are needed to determine the sustained efficacy of these drugs.

Keywords: Antibodies, Monoclonal, Humanized; Choroidal Neovascularization; Intravitreal Injections; Myopia, Degenerative; Bevacizumab; Ranibizumab; Visual Acuity.

\section{INTRODUÇÃO}

A miopia patológica (MP) é uma das principais causas de cegueira nos países desenvolvidos, com uma prevalência na população geral que ronda os $2 \%{ }^{1,2}$ Define-se como um erro refrativo de pelo menos -6 dioptrias, acompanhada de alterações degenerativas da retina, esclera e coróide típicas. ${ }^{3-5}$ Cerca de $10 \%$ dos doentes desenvolvem neovascularização coroideia (NVC), a mais frequente complicação associada e que pode ser responsável pela perda severa e progressiva de visão, sobretudo em jovens e adultos de meia-idade na fase mais produtiva das suas vidas. ${ }^{6-8}$ Ainda que o curso natural da neovascularização coroideia seja variado, quando não tratada está associada a um prognóstico desfavorável. ${ }^{9,10}$

O processo de angiogénese a nível ocular envolve inú-

1. Departamento de Oftalmologia. Hospital de São João/Faculdade de Medicina. Universidade do Porto. Porto. Portugal. Recebido: 22 de Fevereiro de 2013 - Aceite: 22 de Dezembro de 2013 | Copyright @ Ordem dos Médicos 2014 
meros mediadores proteicos e bioquímicos, no qual o factor de crescimento endotelial vascular (VEGF) desempenha um papel central e cuja inibição é uma importante estratégia terapêutica. ${ }^{3,11}$ De facto, estudos demonstraram que os níveis de VEGF se encontram aumentados no humor aquoso dos doentes com neovascularização ativa, quer na degenerescência macular da idade (DMI), quer na miopia patológica. ${ }^{12}$

Como opções terapêuticas para a NVC, foram já usadas fotocoagulação a laser, cirurgia submacular, radioterapia e translocação da mácula. Contudo, os resultados obtidos são variáveis, estando associados a elevadas taxas de recorrência e perda visual progressiva, não permitindo, deste modo, estabelecer um benefício global. ${ }^{13-16} \mathrm{~A}$ terapêutica fotodinâmica com verteporfina, aprovada para o tratamento da NVC miópica, permitiu bons resultados na recuperação da visão, sem danos colaterais na restante retina neurosensorial. Contudo, os resultados perdem significado a longo prazo, provavelmente devido ao maior risco de atrofia corioretiniana nestes doentes, bem como à lesão dos fotorreceptores e do epitélio pigmentar da retina (EPR). ${ }^{17-20}$ Surgiu assim a necessidade de introduzir novas terapêuticas, nomeadamente as injecções intravítreas de agentes anti-VEGF, que mostraram melhores resultados no tratamento. ${ }^{4,6,21-25}$

A terapêutica antiangiogénica, nomeadamente o bevacizumab e o ranibizumab, estão aprovados para o tratamento da neovascularização coroideia secundária à degenerescência macular da idade (DMI), tendo também mostrado claros benefícios clínicos na neovascularização associada a outras situações de causa inflamatória ou vascular.,3,26 Face à evidência de eficácia e segurança com o uso destes agentes, a sua utilização na NVC associada à miopia pa-

Tabela 1 - Caraterísticas Clínicas e Demográficas tológica é atualmente considerada terapêutica de primeira linha. ${ }^{2-4,11,26} \mathrm{O}$ ranibizumab e o bevacizumab são anticorpos monoclonais antiangiogénicos utilizados no tratamento da neovascularização coroideia, com resultados favoráveis a curto prazo. ${ }^{9,27}$ Estudos recentes demonstraram resultados benéficos e sustentados da terapêutica com bevacizumab na NVC miópica, ${ }^{28,29}$ estando em curso ensaios clínicos randomizados e multicêntricos para avaliar a eficácia do ranibizumab a longo prazo.

Os objetivos deste estudo são avaliar os resultados a longo prazo do tratamento antiangiogénico, com ranibizumab e/ou bevacizumab, na NVC associada à miopia patológica, e caracterizar os factores preditivos para os resultados funcionais e anatómicos finais.

\section{MATERIAL E MÉTODOS}

Realizámos uma análise retrospectiva dos registos clínicos dos doentes com NVC miópica tratados com injecções intravítreas de antiangiogénicos no Serviço de Oftalmologia do Hospital de São João entre Janeiro de 2007 e Outubro de 2012.

Os critérios de inclusão foram: miopia com um erro refractivo de equivalente esférico $>-6,0$ dioptrias, em olhos fáquicos, ou comprimento axial $\geq 26,5 \mathrm{~mm}$, em olhos pseudofáquicos ou afáquicos; NVC sub-foveal, justa-foveal (1 a $199 \mu \mathrm{m}$ do centro da área avascular central) ou extrafoveal (além dos $200 \mu \mathrm{m}$ ); doença ativa com difusão na angiografia fluoresceínica (AF); com ou sem tratamento prévio; período mínimo de 12 meses de seguimento. A presença de NVC ativa foi determinada com base nas características da angiografia fluoresceínica (AF) e da tomografia óptica computorizada (OCT).

Os critérios de exclusão foram os seguintes: NVC

Idade (anos)

$57,1 \pm 16,2$

Género (M/F)

Tipo Lesão

Localização Lesão

Sub-foveal 
secundária a outras causas como degenerescência macular da idade (DMI), estrias angióides, coroidite ou trauma; cirurgia intraocular da vitreorretina durante o período de estudo; vasculopatia retiniana como retinopatia diabética ou oclusão venular retiniana; perda de seguimento dos doentes; presença de alergia à fluoresceína.

Avaliámos os resultados de 84 olhos de 81 doentes, dos quais 22 tratados com bevacizumab, 29 tratados com ranibizumab e 33 tratados com ambos os fármacos. Procedemos à recolha de dados relativos à avaliação estereoscópica do fundo ocular, melhor acuidade visual corrigida (MAVC) com escala ETDRS, espessura foveal (EF) na OCT e características da angiografia fluoresceínica, inicial e após 3, 6, 12, 24, 36, 48 e 60 meses de tratamento.

Medimos a MAVC utilizando a escala de ETDRS, de acordo com o protocolo de refracção utilizado no serviço de Oftalmologia do Hospital de São João do Porto. Medimos as espessuras da fóvea utilizando a tomografia de coerência óptica macular: OCT Stratus (Zeiss), versão 4.0.2 e/ou HRA-OCT (Heidelberg Engeneering). $\mathrm{Na}$ angiografia fluoresceínica (AF), avaliámos a difusão de contraste, o crescimento da lesão e definimos lesão precoce, como sendo uma lesão de NVC sem fibrose e ocupando menos de meio disco óptico de área. As lesões de NVC foram classificadas angiograficamente em predominantemente clássicas, minimamente clássicas e ocultas sem componente clássico (Tipo 1). Todos os subtipos de lesão angiográfica foram considerados no estudo, incluindo aqueles com área de fibrose, atrofia ou hemorragia superior a $50 \%$ do tamanho da lesão. Lesões com cicatriz fibrótica sem NVC ativa não foram incluídas no estudo.

As injecções intravítreas de $1.25 \mathrm{mg}$ de bevacizumab (IVB) e/ou $0.5 \mathrm{mg}$ de ranibizumab (IVR) foram administradas a doentes com NVC ativa na angiografia fluoresceínica ou com presença de líquido intra ou subretiniano na OCT. Os doentes não receberam terapêutica combinada com terapia fotodinâmica, triamcinolona ou outro fármaco antiangiogénico. No entanto, não foram excluídos doentes com terapêutica prévia às injecções de anti-VEGF. Todos os doentes incluídos no estudo foram tratados em regime de 1+PRN, consistindo na injecção intravítrea de bevacizumab e/ou ranibizumab, conforme a necessidade, após a primeira injecção.

Para a análise estatística utilizámos o SPSS 20.0. Para as variáveis contínuas utilizámos o Teste t para amostras emparelhadas ou independentes. Utilizámos o Teste de Levene para verificar a homogeneidade da variância. Para avaliar os fatores pré-tratamento preditivos da MAVC final recorremos à análise de regressão linear multivariável. Valores de $p<0,05$ foram considerados estatisticamente significativos.

A aprovação do estudo foi concedida pela Comissão de Ética para a Saúde da Universidade do Porto, estando este de acordo com a Declaração de Helsínquia.

\section{RESULTADOS}

Do total de olhos estudados, 84 (100\%) completaram 12 meses de seguimento, 67 (79,8\%) 24 meses de seguimento, $54(64,3 \%) 36$ meses de seguimento, 29 (34,5\%) 48 meses de seguimento e $15(16,7 \%)$ completaram 60 meses de seguimento. Vinte e nove $(34,5 \%)$ foram tratados apenas com ranibizumab (IVR), $22(26,2 \%)$ apenas com bevacizumab (IVB) e $33(39,3 \%)$ tratados com ambos os fármacos (IVR/IVB). As características clínicas e demográficas dos doentes encontram-se sumarizadas na Tabela 1.

Para o total de olhos estudados, a média da MAVC inicial foi de 43,7 $\pm 20,1$ letras e a média da EF inicial de 304,8 $\pm 127,9 \mu \mathrm{m}$. As diferenças na MAVC inicial, bem como na EF inicial, entre os grupos com diferentes tempos de seguimento não foram estatisticamente significativas $(p=0,8)$.

Avaliámos e comparámos a média da MAVC inicial e da EF inicial, para os doentes com e sem tratamento prévio, e

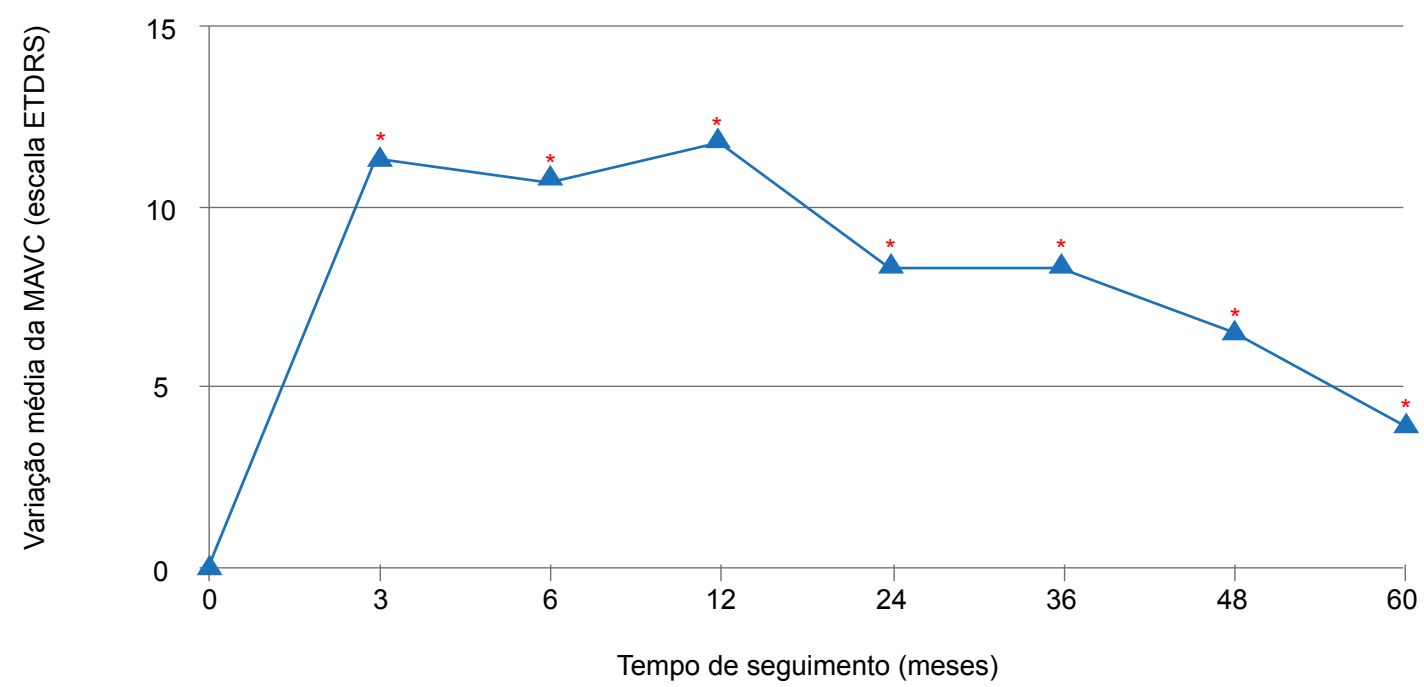

Figura 1 - Variação da média da MAVC relativamente à MAVC inicial. $n=84$ aos 3, 6 e 12 meses; $n=67$ aos 24 meses; $n=54$ aos 36 meses; $n=29$ aos 48 meses; $n=15$ aos 60 meses.

${ }^{*}$ valor $p<0,05$ 
verificámos que doentes submetidos a tratamento prévio, apresentam uma menor EF inicial $(230,4 \pm 95,9 \mu \mathrm{m})$, comparativamente aos não tratados $(308,3 \pm 1259 \mu \mathrm{m})$, sendo essa diferença estatisticamente significativa $(p=0,04)$. Não encontrámos diferenças significativas relativamente à MAVC inicial (45,9 $\pm 19,8$ e 43,8 $\pm 20,2$, respetivamente), $p=0,8$. Avaliámos ainda se a localização da lesão teria impacto nas MAVC e EF iniciais, no entanto as diferenças encontradas não foram estatisticamente significativas ( $p=0,2$ e $p=0,8$, respetivamente).

Após o tratamento com as injecções intravítreas, a média da MAVC foi de $55,1 \pm 17,9$ letras para os três meses de tratamento, $54,5 \pm 18,9$ para os seis meses, $55,6 \pm 18,5$ para os 12 meses, $52,1 \pm 22,3$ para os 24 meses, $52,1 \pm$ 22,6 para os 36 meses, $50,3 \pm 23,8$ para os 48 meses e $47,8 \pm 24,5$ para os 60 meses. A melhoria na MAVC final relativamente à inicial foi estatisticamente significativa para os diferentes tempos de seguimento $(p<0,05)$. A Fig. 1 mostra a variação da MAVC relativamente aos valores iniciais.

Ajustámos os resultados da MAVC às variáveis pré-tratamento, e verificámos que a MAVC inicial se correlaciona de forma independente com melhores resultados na MAVC final $(p<0,001)$ (Tabela 2).

A média das EF foi de $227,9 \pm 98,9$ para os três meses, $224,8 \pm 84,9$ para os seis meses, $209,7 \pm 86,2$ para os 12 meses, 190,6 $\pm 76,1$ para os 24 meses, $174,7 \pm 60,6$ para os 36 meses, $189,8 \pm 96,7$ para os 48 meses e 159,4 \pm 73,3 para os 60 meses. A variação da EF relativamente aos valores iniciais foi estatisticamente significativa para todos os tempos de seguimento (Fig. 2).

O número médio de injecções foi de $5,1 \pm 2,6$ aos 12 meses, $2,1 \pm 2,6$ entre os 12 e os 24 meses, $1,9 \pm 2,5$ entre os 24 e os 36 meses, $1,8 \pm 2,5$ entre os 36 e os 48 meses e 1,2 $\pm 2,2$ entre os 48 e os 60 meses. A redução gradual do número de injecções foi estatisticamente significativa $(p<0,001)$.

Em 81 dos 84 olhos, avaliámos a relação entre a presença de lesão precoce e o número de injecções. O número médio de injecções no grupo de doentes com lesão precoce foi inferior aos doentes com lesão tardia $(8,1 \pm 8,9 \mathrm{e}$ $9,9 \pm 10,4$, respetivamente). No entanto, estas diferenças não foram estatisticamente significativas $(p=0,2)$. Avaliámos ainda se a presença de lesão precoce estaria relacionada com um menor crescimento da lesão a longo prazo, definido pelo aumento de espessura na OCT. Dos 28 $(34,6 \%)$ doentes com lesão precoce, 19 (67,9\%) não tiveram crescimento da lesão ao longo do tempo de seguimento e em nove $(32,1 \%)$ doentes a lesão cresceu. De igual modo, dos $33(40,7 \%)$ doentes em que a lesão cresceu, nove $(27,3 \%)$ apresentavam lesão precoce, e $24(72,7 \%)$ apresentavam lesão tardia. No entanto, nenhum dos resultados foi estatisticamente significativo $(p=0,3)$.

Para o total de doentes, avaliámos ainda separadamente os resultados da MAVC e da EF para os $29(34,5 \%)$ doentes com idade igual ou inferior a 50 anos e para os 55 $(65,5 \%)$ doentes com idade superior a 50 anos (Tabela 3 ). 


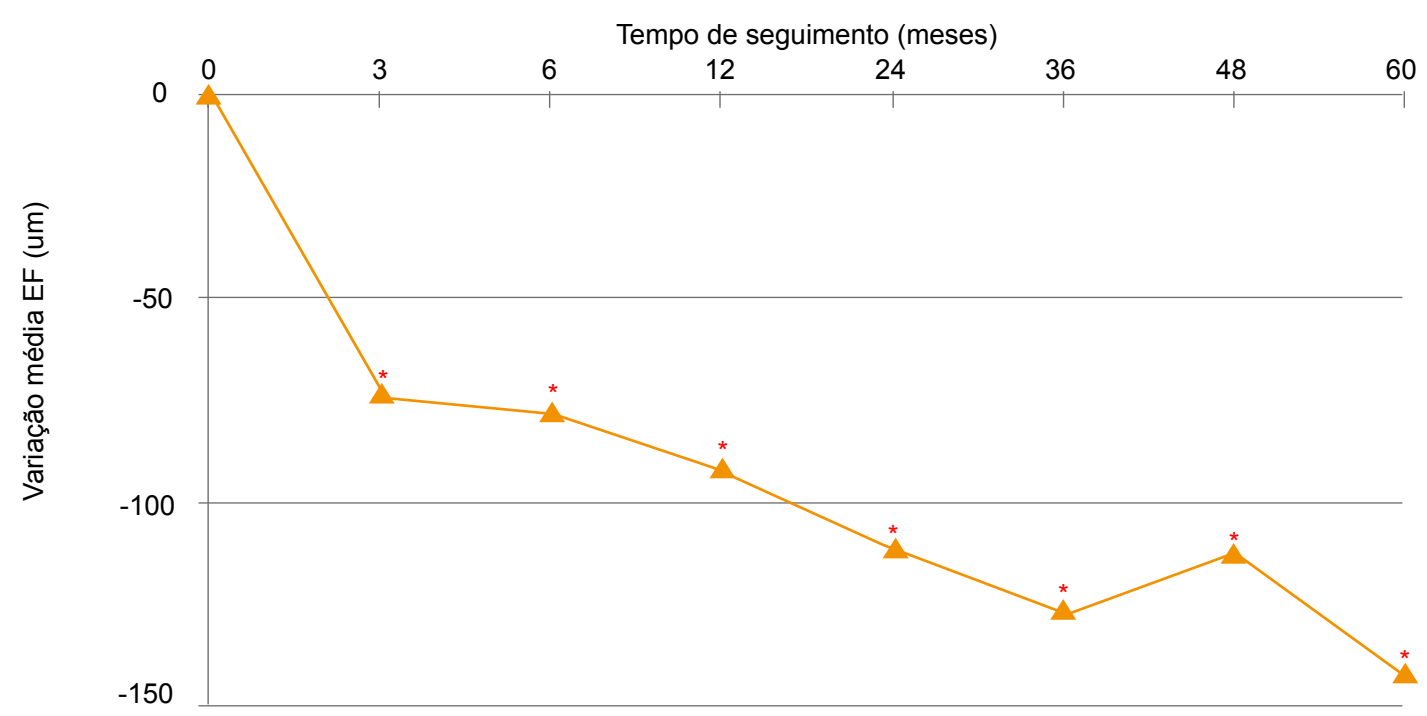

Figura 2 - Variação da EF relativamente à EF inicial. $n=84$ aos 3, 6 e 12 meses; $n=67$ aos 24 meses; $n=54$ aos 36 meses; $n=29$ aos 48 meses; $n=15$ aos 60 meses.

${ }^{*}$ valor $p<0,05$

As diferenças na MAVC e EF iniciais entre os dois grupos não foram estatisticamente significativas $(p=0,3$ e $p=0,4$, respetivamente). As diferenças na MAVC para os diferentes tempos de seguimento não foram estatisticamente significativas entre os dois grupos ( $p=0,9$ aos 3 e 6 meses; $p=0,7$ aos 12 meses; $p=0,4$ aos 24 meses; $p=0,2$ aos 36 meses; $p=0,3$ aos 48 meses e $p=0,5$ aos 60 meses). Relativamente às $E F$, encontrámos diferenças estatisticamente significativas aos 12, 24 e 60 meses, verificando-se uma menor EF no grupo com idade superior a 50 anos ( $p=$ $0,03, p=0,03$ e $p=0,01$ respetivamente). Aos 3, 6, 36 e 48 meses as diferenças não foram significativas $(p=0,3$ aos três meses; $p=0,6$ aos seis meses; $p=0,1$ aos 36 meses e $p=0,2$ aos 48 meses).

Relativamente a complicações do tratamento, uma doente desenvolveu uma reacção inflamatória (vitrite) ao bevacizumab. Não se verificaram complicações sistémicas graves ou outras complicações oculares, nomeadamente endoftalmite, hemorragia vítrea, descolamento da retina, cataratas ou glaucoma, durante o período de seguimento.

\section{Análise dos subgrupos de doentes tratados com bevacizumab elou ranibizumab}

As médias das MAVC e EF nos diferentes grupos, para os diferentes tempos de seguimento estão representadas na Tabela 4. No grupo tratado com ranibizumab, as diferenças encontradas na MAVC relativamente aos valores iniciais foram estatisticamente significativas para os $3,6 \mathrm{e}$ 12 meses $(p<0,05)$. A partir dos 24 meses de seguimento, as diferenças não foram estatisticamente significativas ( $p=0,3$ aos 24 meses; $p=0,1$ aos 36 meses; $p=0,1$ aos 48 meses e $p=0,2$ aos 60 meses). Relativamente à $E F$, as diferenças encontradas relativamente aos valores iniciais são estatisticamente significativas para os $3,6,12,24$ e
36 meses $(p<0,05)$, deixando de o ser para tempos de seguimento mais longos ( $p=0,1$ aos 48 meses e $p=1$ aos 60 meses).

Relativamente ao tratamento com bevacizumab, verificámos que as diferenças na MAVC final relativamente à inicial são estatisticamente significativas até tempos de seguimento de 36 meses, não o sendo para os 48 e 60 meses de seguimento ( $p=0,1$ e $p=0,4$, respetivamente). A diferença da $E F$ final comparativamente à inicial é estatisticamente significativa para os $3,6,12,36$ e 60 meses $(p<0,05)$. Para os 24 e 48 meses, as diferenças entre os parâmetros não são estatisticamente significativas $(p=0,2$ e $p=0,1$, respetivamente).

Nos doentes tratados com ambos os fármacos, verificámos, para os diferentes tempos de seguimento, que a diferença entre a MAVC final e a inicial foi estatisticamente significativa até aos 36 meses de seguimento, não o sendo aos 48 e aos 60 meses ( $p=0,9$ e $p=0,1$, respetivamente). A EF final é menor comparativamente à inicial, sendo as diferenças estatisticamente significativas até as 48 meses de seguimento, mas não para os 60 meses de seguimento $(p=0,1)$.

Comparámos os valores da MAVC entre os diferentes grupos, para os mesmos tempos de seguimento, mas não encontrámos diferenças estatisticamente significativas $(p=0,7 ; p=0,9 ; p=0,8 ; p=0,8 ; p=0,8 ; p=0,8 ; p=0,7$ e $p=0,2$ para os valores iniciais, aos $3,6,12,24,36,48$ e 60 meses de seguimento, respetivamente). A EF aos 60 meses de seguimento apresenta melhores resultados para o grupo tratado com bevacizumab $(p=0,02)$. Não foram encontradas diferenças na EF estatisticamente significativas para os restantes tempos de seguimento $(p=0,5 ; p=0,6$; $p=0,7 ; p=0,9 ; p=0,2 ; p=0,1$ para os $3,6,12,24,36$ e 48 meses de seguimento, respetivamente). 
Tabela 3 - Média da MAVC e EF para os diferentes tempos de seguimento em função da idade ( $\leq 50$ anos e > 50 anos)

\begin{tabular}{|c|c|c|c|c|c|c|c|c|}
\hline & \multicolumn{8}{|c|}{$\begin{array}{c}\text { Idade } \leq 50 \text { anos } \\
(n=29)\end{array}$} \\
\hline & $\begin{array}{l}\text { Inicial } \\
(n=29)\end{array}$ & $\begin{array}{l}\text { 3meses } \\
(n=29)\end{array}$ & $\begin{array}{l}6 \text { meses } \\
(n=29)\end{array}$ & $\begin{array}{c}12 \text { meses } \\
(n=29)\end{array}$ & $\begin{array}{c}24 \text { meses } \\
(n=21)\end{array}$ & $\begin{array}{l}36 \text { meses } \\
(n=18)\end{array}$ & $\begin{array}{c}\text { 48meses } \\
(n=9)\end{array}$ & $\begin{array}{c}60 \text { meses } \\
(n=6)\end{array}$ \\
\hline $\begin{array}{l}\text { MAVC } \\
\text { (letras) }\end{array}$ & $46,9 \pm 20,2$ & $55,4 \pm 17,7$ & $55,5 \pm 18,2$ & $56,9 \pm 18,3$ & $56,7 \pm 18,4$ & $58,1 \pm 20,7$ & $58,1 \pm 18,9$ & $54,6 \pm 21,9$ \\
\hline \multirow[t]{3}{*}{$\begin{array}{c}E F \\
(\mu \mathrm{m})\end{array}$} & $282,8 \pm 94,1$ & $230,9 \pm 73,9$ & $225,7 \pm 72,3$ & $225,1 \pm 70,3$ & $208,2 \pm 43,4$ & $190,8 \pm 39,9$ & $\begin{array}{c}234,6 \pm \\
130,4\end{array}$ & $208,2 \pm 49,5$ \\
\hline & \multicolumn{8}{|c|}{$\begin{array}{c}\text { Idade }>50 \text { anos } \\
(n=55)\end{array}$} \\
\hline & $\begin{array}{l}\text { Inicial } \\
(n=55)\end{array}$ & $\begin{array}{l}3 \text { meses } \\
(n=55)\end{array}$ & $\begin{array}{l}\text { 6meses } \\
(n=55)\end{array}$ & $\begin{array}{l}12 \text { meses } \\
(n=55)\end{array}$ & $\begin{array}{c}24 \text { meses } \\
(n=46)\end{array}$ & $\begin{array}{c}36 \text { meses } \\
(n=36)\end{array}$ & $\begin{array}{l}\text { 48meses } \\
(n=20)\end{array}$ & $\begin{array}{c}\text { 60meses } \\
(n=9)\end{array}$ \\
\hline $\begin{array}{l}\text { MAVC } \\
\text { (letras) }\end{array}$ & $42 \pm 19,9$ & $54,6 \pm 18,9$ & $53,6 \pm 20,3$ & $54,6 \pm 19,6$ & $49,5 \pm 24,6$ & $48,5 \pm 24,3$ & $46,8 \pm 25,4$ & $44 \pm 26,2$ \\
\hline $\begin{array}{c}\text { EF } \\
(\mu \mathrm{m})\end{array}$ & $316,11 \pm 41,8$ & $223,4 \pm 107,9$ & $222,4 \pm 91,7$ & $200,7 \pm 92,5$ & $181,3 \pm 87,3$ & $168,5 \pm 67,4$ & $156,5 \pm 61,2$ & $123,4 \pm 54,8$ \\
\hline
\end{tabular}

EF, Espessura foveal ( $\mu \mathrm{m})$; MAVC, Melhor acuidade visual corrigida (escala ETDRS).

\section{DISCUSSÃO}

Quando não tratada, a miopia patológica apresenta mau prognóstico, estando associada a perda de visão severa e progressiva. ${ }^{9,10,30}$ Deste modo, e tendo em conta os resultados não sustentados com a terapia fotodinâmica, os novos fármacos antiangiogénicos têm sido cada vez mais utilizados no tratamento de patologias que cursam com NVC, como é o caso da miopia patológica.

O ranibizumab e o bevacizumab são anticorpos monoclonais anti-VEGF utilizados no tratamento da neovascularização coroideia, pelo seu efeito na redução da proliferação celular, da permeabilidade vascular e da formação de novos vasos sanguíneos. ${ }^{9,12,31}$ A sua eficácia e segurança estão descritas em vários estudos retrospectivos e em alguns ensaios clínicos prospectivos. Assim, temos assistido ao uso difundido destes agentes no tratamento da neovascularização coroideia associada tanto à MP como à DMI, com melhorias funcionais e anatómicas significativas. ${ }^{32} \mathrm{O}$ ranibizumab corresponde a um fragmento Fab monoclonal, humanizado, recombinante, com um peso molecular de 49 $\mathrm{kDa} .{ }^{33}$ Desenvolvido especificamente para uso intraocular, apresenta algumas vantagens teóricas relativamente ao bevacizumab, nomeadamente o menor peso molecular, associado a uma melhor e mais rápida penetração nas camadas da retina, maior afinidade para o recetor VEGF-A e menor incidência de efeitos sistémicos. ${ }^{12} \mathrm{O}$ bevacizumab é um anticorpo monoclonal, humanizado, recombinante, ${ }^{33}$ desenvolvido para tratamento endovenoso, como adjuvante da quimioterapia, na terapia de neoplasias sólidas metastizadas. Assume particular interesse no tratamento da NVC na miopia, pelos resultados promissores descritos em séries de casos e alguns ensaios clínicos, 1,6,8,11,13,34-36 aliados a um preço significativamente mais baixo quando comparado com o ranibizumab, permitindo deste modo que a terapêutica seja acessível a um maior número de doentes. ${ }^{10}$

Verificámos que os agentes antiangiogénicos permitiram uma melhoria significativa e sustentada na MAVC e na EF, até aos 60 meses de tratamento. Os nossos resultados estão de acordo com outros descritos para o ranibizumab $^{37-41}$ e para o bevacizumab em estudos com menor tempo de seguimento. ${ }^{36,42} A$ variação da MAVC relativamente ao valor inicial foi progressivamente menor para os diferentes tempos de seguimento, embora sempre superiores à MAVC inicial $(p<0,05)$, o que poderá ser explicado pela idade mais avançada, com componente associado de DMI e consequentemente MAVC mais baixas, bem como pela progressão da atrofia associada à miopia e à própria NVC. Por sua vez, a variação na EF foi progressivamente maior, o que poderá estar relacionado não só com a redução do edema macular, mas também com a atrofia corioretiniana nos doentes miópicos.

Os doentes submetidos a terapêutica prévia apresentaram menor espessura da fóvea inicial comparativamente 
Tabela 4 - Média da MAVC e EF para os diferentes tempos de seguimento, em função do tratamento com ranibizumab e/ou bevacizumab

\begin{tabular}{|c|c|c|c|c|c|c|c|c|c|c|c|c|}
\hline & & $\begin{array}{l}\text { Inicial } \\
(n=84)\end{array}$ & & & $\begin{array}{c}3 \text { meses } \\
(n=84)\end{array}$ & & & $\begin{array}{c}6 \text { meses } \\
(n=84)\end{array}$ & & & $\begin{array}{c}12 \text { meses } \\
(n=84)\end{array}$ & \\
\hline & $\begin{array}{c}\text { IVR } \\
(n=29)\end{array}$ & $\begin{array}{c}\text { IVB } \\
(n=22)\end{array}$ & $\begin{array}{l}\text { IVR/IVB } \\
(n=33)\end{array}$ & $\begin{array}{c}\text { IVR } \\
(n=29)\end{array}$ & $\begin{array}{c}\text { IVB } \\
(n=22)\end{array}$ & $\begin{array}{l}\text { IVR/IVB } \\
(n=33)\end{array}$ & $\begin{array}{c}\text { IVR } \\
(n=29)\end{array}$ & $\begin{array}{c}\text { IVB } \\
(n=22)\end{array}$ & $\begin{array}{l}\text { IVR/IVB } \\
(n=33)\end{array}$ & $\begin{array}{c}\text { IVR } \\
(n=29)\end{array}$ & $\begin{array}{c}\text { IVB } \\
(n=22)\end{array}$ & $\begin{array}{l}\text { IVR/IVB } \\
(n=33)\end{array}$ \\
\hline $\begin{array}{l}\text { MAVC } \\
\text { (letras) }\end{array}$ & $\begin{array}{c}46,2 \\
\pm \\
22,1\end{array}$ & $\begin{array}{c}41,6 \\
\pm \\
20,4\end{array}$ & $\begin{array}{c}42,9 \\
\pm \\
18,2\end{array}$ & $\begin{array}{c}53,8 \\
\pm \\
22,9\end{array}$ & $\begin{array}{c}55,9 \\
\pm \\
16,5\end{array}$ & $\begin{array}{c}55 \\
\pm \\
15,5\end{array}$ & $\begin{array}{c}53,7 \\
\pm \\
21,8\end{array}$ & $\begin{array}{c}55,6 \\
\pm \\
20,8\end{array}$ & $\begin{array}{c}53,8 \\
\pm \\
26,8\end{array}$ & $\begin{array}{c}52,7 \\
\pm \\
23,2\end{array}$ & $\begin{array}{c}54,7 \\
\pm \\
17,3)\end{array}$ & $\begin{array}{c}58,1 \\
\pm \\
16,1\end{array}$ \\
\hline $\begin{array}{c}\text { EF } \\
(\mu \mathrm{m})\end{array}$ & $\begin{array}{c}284,9 \\
\pm \\
121,3\end{array}$ & $\begin{array}{c}307,3 \\
\pm \\
146,1\end{array}$ & $\begin{array}{c}321,3 \\
\pm \\
124,5\end{array}$ & $\begin{array}{c}204,7 \\
\pm \\
61,3\end{array}$ & $\begin{array}{c}258,4 \\
\pm \\
130,7\end{array}$ & $\begin{array}{c}225,1 \\
\pm \\
96,8\end{array}$ & $\begin{array}{c}205,6 \\
\pm \\
56,6\end{array}$ & $\begin{array}{c}236,2 \\
\pm \\
101,6\end{array}$ & $\begin{array}{c}231,7 \\
\pm \\
94,1\end{array}$ & $\begin{array}{c}207,5 \\
\pm \\
77,1\end{array}$ & $\begin{array}{c}207,1 \\
\pm \\
99,7\end{array}$ & $\begin{array}{c}212,4 \\
\pm \\
86,4\end{array}$ \\
\hline
\end{tabular}

Tabela 4 - Média da MAVC e EF para os diferentes tempos de seguimento, em função do tratamento com ranibizumab e/ou bevacizumab (continuação)

\begin{tabular}{|c|c|c|c|c|c|c|c|c|c|c|c|c|}
\hline & & $\begin{array}{c}24 \text { meses } \\
(n=67)\end{array}$ & & & $\begin{array}{c}36 \text { meses } \\
(n=54)\end{array}$ & & & $\begin{array}{c}48 \text { meses } \\
(n=29)\end{array}$ & & & $\begin{array}{c}60 \text { meses( } \\
n=14)\end{array}$ & \\
\hline & $\begin{array}{c}\text { IVR } \\
(n=25)\end{array}$ & $\begin{array}{c}\text { IVB } \\
(n=12)\end{array}$ & $\begin{array}{l}\text { IVR/IVB } \\
(n=30)\end{array}$ & $\begin{array}{c}\text { IVR } \\
(n=18)\end{array}$ & $\begin{array}{c}\text { IVB } \\
(n=12)\end{array}$ & $\begin{array}{l}\text { IVR/IVB } \\
(n=24)\end{array}$ & $\begin{array}{c}\text { IVR } \\
(n=4)\end{array}$ & $\begin{array}{c}\text { IVB } \\
(n=12)\end{array}$ & $\begin{array}{l}\text { IVR/IVB } \\
(n=13)\end{array}$ & $\begin{array}{c}\text { IVR } \\
(n=2)\end{array}$ & $\begin{array}{c}\text { IVB } \\
(n=10)\end{array}$ & $\begin{array}{c}\text { IVR/IVB } \\
(n=3)\end{array}$ \\
\hline $\begin{array}{l}\text { MAVC } \\
\text { (letras) }\end{array}$ & $\begin{array}{c}50 \\
\pm \\
24,1\end{array}$ & $\begin{array}{c}49,2 \\
\pm \\
25,5\end{array}$ & $\begin{array}{c}54,3 \\
\pm \\
21,4\end{array}$ & $\begin{array}{c}53,8 \\
\pm \\
24,4\end{array}$ & $\begin{array}{c}49,2 \\
\pm \\
24,9\end{array}$ & $\begin{array}{c}51,3 \\
\pm \\
22,9\end{array}$ & $\begin{array}{c}57,5 \\
\pm \\
25,6\end{array}$ & $\begin{array}{c}49,7 \\
\pm \\
25,4\end{array}$ & $\begin{array}{c}48,6 \\
\pm \\
23,4\end{array}$ & $\begin{array}{c}45 \\
\pm \\
38,2\end{array}$ & $\begin{array}{c}41,7 \\
\pm \\
23,3\end{array}$ & $\begin{array}{c}68 \\
\pm \\
14,2\end{array}$ \\
\hline $\begin{array}{c}\text { EF } \\
(\mu \mathrm{m})\end{array}$ & $\begin{array}{c}176,9 \\
\pm \\
64\end{array}$ & $\begin{array}{c}160 \\
\pm \\
67,1\end{array}$ & $\begin{array}{c}212 \\
\pm \\
84,5\end{array}$ & $\begin{array}{c}193,2 \\
\pm \\
54,2\end{array}$ & $\begin{array}{c}139,8 \\
\pm \\
54,9\end{array}$ & $\begin{array}{c}179,9 \\
\pm \\
61,7\end{array}$ & $\begin{array}{c}218,3 \\
\pm \\
50,9\end{array}$ & $\begin{array}{c}135,4 \\
\pm \\
59,5\end{array}$ & $\begin{array}{c}210,6 \\
\pm \\
114\end{array}$ & $\begin{array}{c}213 \\
\pm \\
67,9\end{array}$ & $\begin{array}{c}115,8 \\
\pm \\
45,3\end{array}$ & $\begin{array}{c}225,3 \\
\pm \\
6,1\end{array}$ \\
\hline
\end{tabular}

EF, Espessura foveal ( $\mu \mathrm{m})$; MAVC, Melhor acuidade visual corrigida (escala ETDRS).

aos que não foram tratados previamente, provavelmente relacionada com a redução do edema macular e da NVC $(230,4 \pm 95,9 \mu$ e 308,3 \pm 125,9 $\mu$, respetivamente; $p=0,04) \cdot{ }^{17,18}$ No entanto, e corroborando os estudos de Calvo-Gonzalez et al, ${ }^{15}$ Monés JM et al ${ }^{39}$ e Lalloum $\mathrm{F}$ et al, ${ }^{41}$ não encontrámos diferenças estatisticamente significativas na acuidade visual inicial de doentes com ou sem tratamento prévio $(45,9 \pm 19,8$ e 43,8 \pm 2,02 letras, respetivamente; $p=0,7)$. Sendo que a menor espessura inicial não se acompanha de uma melhor acuidade visual significativa, esta poderá ser explicada pela progressão da atrofia corioretiniana a que a TFD predispõe..$^{17}$ Por outro lado, sabemos que os resultados desta terapêtica estão demonstrados apenas a curto prazo, o que pode justificar o facto de, aquando do início do tratamento antiangiogénico, não existirem já diferenças na acuidade visual entre os grupos.

Está descrito que a NVC não subfoveal pode ser fator preditor de melhor prognóstico, uma vez que não afeta diretamente a zona central. ${ }^{4,13,43}$ No nosso estudo 69 dos 84 olhos $(81,1 \%)$ apresentavam lesão subfoveal. No entanto, a localização da lesão não influenciou significativamente a MAVC ou a EF ( $p=0,2$ e $p=0,8$, respetivamente).

$A$ acuidade visual pré-tratamento influenciou positi- vamente a acuidade visual a longo-prazo $(p<0,001)$. De facto, uma melhor MAVC inicial está associada a menos dano nos fotorreceptores, que poderá explicar os melhores resultados. ${ }^{15,25}$ No entanto, outros estudos mostram que uma MAVC inicial mais baixa cursa com melhores resultados após tratamento, ${ }^{13}$ de acordo com o ceiling floor effect. Os autores consideram que uma melhor MAVC inicial terá menor probabilidade de melhorar (ceiling effect), enquanto doentes com pior MAVC inicial terão mais probabilidade de melhorar (floor effect).

A terapêutica prévia não influenciou significativamente os resultados da MAVC final $(p>0,05)$. Alguns estudos demonstram que olhos sem terapêutica prévia têm melhores resultados com o tratamento anti-VEGF. ${ }^{15,24}$ Nos doentes previamente tratados, presumivelmente com maior curso da doença e com recorrência da NVC, poderá haver mais resistência ao tratamento antiangiogénico, o que justificaria os piores resultados. ${ }^{8,15}$ Acresce ainda o facto de a TFD predispor à trombose dos vasos da coróide e à atrofia corioretiniana. ${ }^{11}$ Estão também descritos melhores resultados em doentes com terapêutica prévia, possivelmente subjacente à perceção precoce dos sintomas que poderá ter levado ao tratamento mais atempado das recorrências. ${ }^{8}$ No entanto, 
nesse estudo o tamanho reduzido da amostra $(n=21)$ não foi suficiente para que os resultados fossem extrapolados.

Como seria de esperar, tendo em conta a menor gravidade da lesão precoce, verificámos que na maioria destes doentes a lesão não cresceu $(67,9 \%)$. No entanto, os resultados não foram estatisticamente significativos, provavelmente devido ao reduzido tamanho da amostra $(p=0,3)$.

Apesar de a idade ser um importante fator prognóstico, com influência na história natural da doença após tratamento com anti VEGF, ${ }^{10,}{ }^{36}$ não foi associada de forma significativa aos resultados da acuidade visual $(p>0,05)$. Os nossos achados podem dever-se ao facto de a maioria dos doentes ter mais de 50 anos $(65,5 \%)$, com idade média de 57,2 (+-16,1). Estes resultados são corroborados por um outro estudo em que o outcome visual dos doentes com idade igual ou inferior a 50 anos foi semelhante àqueles com mais de $50 .{ }^{44}$ Os doentes com mais de 50 anos apresentaram uma menor EF final para os diferentes tempos de seguimento, tendência que foi significativa para os 12, 24 e 60 meses de seguimento $(p=0,03, p=0,03$ e $p=0,001$, respetivamente). Isto poderá provavelmente estar relacionado com a redução do edema após o tratamento antiangiogénico. Contudo, devemos também considerar a hipótese da menor EF final ser devida à atrofia corioretiniana progressiva nos doentes míopes, sobretudo em idades mais avançadas. Acresce ainda o facto de podermos estar perante um componente misto de MP e DMI, que agrava a atrofia e justifica a menor EF nestes doentes. Sabe-se que o aumento da atrofia corioretiniana ao longo dos anos está associado a pior prognóstico em doentes com NVC miópica e pode ser devido à progressão natural da doença e ao impacto negativo do tratamento antiangiogénico ${ }^{45}$ ou do tratamento prévio. ${ }^{15}$

Tendo em conta as diferenças moleculares acima descritas entre o ranibizumab e o bevacizumab, sugeriu-se uma diferença na eficácia clínica destes dois fármacos na MP. ${ }^{46}$ Contudo, verificámos melhorias semelhantes na acuidade visual entre os diferentes grupos de tratamento. Os nossos resultados são consistentes com o estudo de Gharbiya et al, ${ }^{9}$ prospectivo, randomizado e controlado, que de igual modo não encontrou diferenças significativas entre a MAVC dos grupos tratados com bevacizumab e/ou ranibizumab, para um tempo de seguimento máximo de seis meses. Neste estudo, a MAVC aos seis meses foi de 43,8 $\pm 9,9$ letras para o grupo tratado com ranibizumab $(n=16)$ e 45,4 \pm 9,9 letras para o grupo tratado com bevacizumab $(n=16)$. No nosso estudo, e para o mesmo tempo de seguimento, a MAVC foi de 53,7 $\pm 21,8$ e 55,6 $\pm 20,8$ para o grupo tratado com ranibizumab $(n=29)$ e bevacizumab $(n=22)$, respetivamente. No entanto, os melhores resultados do nosso estudo estão provavelmente relacionados com a melhor MAVC inicial $(46,2 \pm 22,1$ no grupo tratado com IVR e 41,6 $\pm 20,4$ no grupo tratado com IVB), comparativamente ao estudo de Gharbiya et al $(26,44 \pm 12,58$ no grupo tratado com IVR e 29,50 $\pm 12,98$ no grupo tratado com IVB). Para além deste, outros estudos corroboram os nossos resultados..$^{40,47,48}$ No entanto, contrariamente aos mesmos, que não encontraram diferenças na EF entre os grupos, verificámos que a EF final no grupo tratado com bevacizumab foi melhor para os 60 meses de seguimento $(115,8 \pm 45,3 \mu \mathrm{m}$ no grupo com IVB, $213 \pm 67,9 \mu \mathrm{m}$ no grupo com IVR e 225,3 \pm 6,1 $\mu \mathrm{m}$ no grupo com IVB/IVR), o que poderá dever-se ao maior peso molecular do fármaco, e consequentemente à maior duração de acção intravítrea. Contudo, dos 15 doentes com 60 meses de seguimento, 10 foram tratados apenas com bevacizumab, o que poderá justificar os melhores resultados neste grupo. As melhorias na MAVC e na EF no grupo tratado apenas com ranibizumab foram significativas até tempos de seguimento mais curtos, o que pode estar subjacente à menor duração de acção comparativamente ao bevacizumab.

No nosso estudo, o tratamento foi baseado no regime $1+P R N$, consistindo na injecção intravítrea de bevacizumab e/ou ranibizumab, conforme a necessidade, após a primeira injecção. Verificámos que os benefícios funcionais e anatómicos foram alcançados a par de uma redução significativa no número de injeções/ano, provavelmente subjacente a uma melhoria desde o início do tratamento. Não verificámos complicações oculares ou sistémicas graves, com exceção de uma doente que desenvolveu uma reacção inflamatória (vitrite) ao bevacizumab. A doente foi tratada, mantendo uma AV semelhante à apresentada anteriormente, e passou para tratamento com ranibizumab. Estão descritos na literatura casos de reacções inflamatórias associadas às injecções intravítreas de agentes anti-VEGF. ${ }^{49-51}$ Embora ainda não totalmente esclarecido, admite-se que o mecanismo subjacente possa estar relacionado com uma resposta imunológica a componentes dos fármacos. De facto, o risco parece ser superior com o bevacizumab, quando comparado com o ranibizumab, provavelmente subjacente à porção $\mathrm{Fc}$ adicional e consequentemente ao maior conteúdo proteico. ${ }^{51,52}$ Uma vez que os doentes com miopia patológica apresentam risco aumentado de desenvolverem complicações oculares, é recomendado um exame da retina detalhado e, eventualmente, terapia laser profiláctica para os olhos de alto risco, antes de iniciar as injecções intravítreas. ${ }^{47}$

De entre as limitações do nosso estudo, importa referir o carácter retrospectivo, a ausência de um grupo controlo e o tamanho reduzido da amostra. A comparação dos diversos estudos é, por vezes, limitada, sobretudo pelo diferente tempo de seguimento, metodologia e critérios de tratamento utilizados. Acresce ainda o facto de os resultados serem altamente dependentes das características dos doentes, da duração dos sintomas, da idade, da área de NVC e da MAVC pré-tratamento. Apesar de não termos medido a área da lesão de NVC, verificámos, na AF e na OCT, uma redução no tamanho da NVC na maioria dos olhos tratados $(59,3 \%)$.

\section{CONCLUSÃO}

Os nossos resultados reflectem a prática clínica e mostram que as injecções intravítreas de anti-VEGF, segundo um regime $1+\mathrm{PRN}$, cursam com uma melhoria significativa 
e sustentada no tempo da acuidade visual em doentes com NVC miópica. Importa salientar que os benefícios conseguidos com o tratamento antiangiogénico foram mantidos para tempos de seguimento de até 60 meses, cuja relevância clínica se deve à história natural da doença. Até à data, apenas um número limitado de estudos e com tempos de seguimento demasiado curtos, comparou directamente os dois fármacos utilizando o mesmo regime de tratamento, sem que fossem encontradas diferenças significativas. Deste modo, a relação custo-benefício do ranibizumab comparativamente ao bevacizumab permanece controversa.

Consideramos que ensaios clínicos multicêntricos, prospectivos, randomizados e com maior número de doen- tes serão necessários para confirmar os resultados do tratamento antiangiogénico a longo prazo, bem como para estabelecer conclusões sobre aspectos de eficácia e segurança, cruciais na escolha do tratamento.

\section{CONFLITOS DE INTERESSE}

Os autores declaram a inexistência de conflitos de interesse na realização do presente trabalho.

\section{FONTES DE FINANCIAMENTO}

Não existiram fontes externas de financiamento para a realização deste artigo.

\section{REFERÊNCIAS}

1. Ruiz-Moreno JM, Montero JA, Amat-Peral P. Myopic choroidal neovascularization treated by intravitreal bevacizumab: comparison of two different initial doses. Graefes Arch Clin Exp Ophthalmol. 2011;249:595-9.

2. Neelam K, Cheung CM, Ohno-Matsui K, Lai TY, Wong TY. Choroidal neovascularization in pathological myopia. Prog Retin Eye Res. 2012;31:495-525.

3. Mitry D, Zambarakji H. Recent trends in the management of maculopathy secondary to pathological myopia. Graefes Arch Clin Exp Ophthalmol. 2012;50:3-13.

4. Silva R. Myopic maculopaty: a review. Ophtalmologica. 2012;228:197213.

5. Moussa S, Ansari-Shahrezaei S, Smretschnig E, Hagen S, SteindlKuscher K, Krebs I, et al. Contrast sensitivity after intravitreal antivascular endothelial growth factor therapy for myopic choroidal neovascularization. Graefes Arch Clin Exp Ophthalmol. 2009;248:1087-90.

6. Parodi MB, lacono P, Papayannis A, Sheth S, Bandello F. Laser photocoagulation, photodynamic therapy, and intravitreal bevacizumab for the treatment of juxtafoveal choroidal neovascularization secondary to pathologic myopia. Arch Ophthalmol. 2010;128:437-42.

7. Ng DS, Kwok AK, Chan CW. Anti-vascular endothelial growth factor for myopic choroidal neovascularization. Clin Exp Ophthalmol. 2012;40:e98-110.

8. Voykov B, Gelisken F, Inhoffen W, Voelker M, Bartz-Schmidt KU, Ziemssen F. Bevacizumab for choroidal neovascularization secondary to pathologic myopia: Is there a decline of the treatment efficacy after 2 years? Graefes Arch Clin Exp Ophthalmol. 2010;248:543-50.

9. Gharbiya M, Giustolisi R, Allievi F, Fantozzi N, Mazzeo L, Scavella V, et al. Choroidal neovascularization in pathologic myopia: Intravitreal ranibizumab versus bevacizumab - a randomized controlled trial. Am J Ophthalmol. 2010;149:458-46.

10. Wang J, Kang Z. Summary of prognostic factors for choroidal neovascularization due to pathological myopia treated by intravitreal bevacizumab injection. Graefes Arch Clin Exp Ophtalmol. 2012;250:1717-23.

11. El Matri L, Kort F, Chebil A, Bouraoui R, Merdassi A, Bouladi M. Intravitreal bevacizumab versus photodynamic therapy for myopic choroidal neovascularization in a North-African population. Graefes Arch Clin Exp Ophthalmol. 2011;249:1287-93.

12. Konstantinidis L, Mantel I, Pournaras JA, Zografos L, Ambresin A. Intravitreal ranibizumab (Lucentis ${ }^{\circledR}$ ) for the treatment of myopic choroidal neovascularization. Graefes Arch Clin Exp Ophthalmol. 2009;247:3118.

13. Bottoni F, Perego E, Airaghi P. Surgical removal of subfoveal choroidal neovascular membranes in high myopia. Graefes Arch Clin Exp Ophthalmol. 1999;237:573-82

14. Ruiz-Moreno JM, de la Vega C. Surgical removal of subfoveal choroidal neovascularization in highly myopic patients. $\mathrm{Br} \mathrm{J}$ Ophthalmol. 2011;85:1041-3

15. Hamelin N, Glacet-Bernard A, Brindeau C. Surgical treatment of subfoveal neovascularization in myopia: macular translocation vs. surgical removal. Am J Ophthalmol. 2002;133:530-6.

16. Uemura A, Thomas MA. Subretinal surgery for choroidal neovascularization in patients with high myopia. Arch Ophtalmol. 2000;118:344-50.

17. Blinder KJ, Blumenkranz MS, Bressler NM, Bressler SB, Donato G, Lewis $\mathrm{H}$ et al. Verteporfin therapy of subfoveal choroidal neovascularization in pathologic myopia: 2-year results of a randomized clinical trial
- VIP report n.3. Ophthalmology. 2003;110:667-73.

18. Verteporfin in Photodynamic Therapy Study Group. Photodynamic therapy of subfoveal choroidal neovascularization in pathologic myopia with verteporfin. 1-year results of a randomized clinical trial - VIP report no.1. Ophthalmology. 2001;108:841-52.

19. Marticorena J, Gomez-Ulla F, Fernandez M, Pazos B, Rodriquez-Cid MJ, Sanchez-Salorio M. Combined photodynamic therapy and intravitreal triamcinolone acetonide for the treatment of myopic subfoveal choroidal neovascularisation. Am J Ophtalmol. 2006;142:335-7.

20. Chan WM, Lai TY, Wong AL, Liu DT, Lam DS. Combined photodynamic therapy and triamcinolone injection for the treatment of choroidal neovascularisation secondary to pathological myopia: a pilot study. $\mathrm{Br} J$ Ophthalmol. 2007;91:131-3.

21. Baba T, Kubota-Taniai M, Kitahashi M, Okada K, Mitamura Y, Yamamoto S. Two year comparison of photodynamic therapy and intravitreal bevacizumab for treatment of myopic choroidal neovascularization. $\mathrm{Br} \mathrm{J}$ Ophthalmolol. 2010;94:864-70.

22. Hayashi K, Ohno-Matsui K, Teramukai S. Comparison of visual outcome and regression pattern of myopic choroidal neovascularization after intravitreal bevacizumab or after photodynamic therapy. Am J Ophthalmol. 2009;148:396-408.

23. Ikuno Y, Nagai Y, Matsuda S, Arisawa A, Sho K, Oshita T. Two-year visual results for older Asian women treated with photodynamic therapy or bevacizumab for myopic neovascularization. Am J Ophtalmol. 2010;149:140-6.

24. Yoon JU, Byun YJ, Koh HJ. Intravitreal anti-VEGF versus photodynamic therapy with verteporfin for treatment of myopic choroidal neovascularization. Retina. 2010;30:418-24.

25. Nakanishi H, Tsujikawa A, Yodoi Y, Ojima Y, Otani A, Tamura H, et al. Prognostic factors for visual outcomes 2-years after intravitreal bevacizumab for myopic choroidal neovascularization. Eye. 2011;25:375-81.

26. Wu PC, Chen YJ. Intravitreal injection of bevacizumab for myopic choroidal neovascularization: 1-year follow-up. Eye. 2009;23:2042-5.

27. Calvo-Gonzalez C, Reche-Frutos J, Donate J, Fernandez-Perez C, Garcia-Feijoo J. Intravitreal ranibizumab for myopic choroidal neovascularization: Factores preditive of visual outcome and need for retreatment. Am J Ophtalmol. 2010;151:529-34.

28. Gharbiya M, Cruciani F, Parisi F, Cuozzo G, Altimari S, Abdolrahimzadeh S. Long-term results of intravitreal bevacizumab for choroidal neovascularisation in pathological myopia. Br J Ophthalmol. 2012;96:1068-72.

29. Peiretti E, Vinci M, Fossarello M. Intravitreal bevacizumab as a treatment for choroidal neovascularization secondary to myopia:4-year study results. Can J Ophthalmol. 2012;47:28-33.

30. Yoshida T, Ohno-Matsui K, Yasuzumi K, Kojima A, Shimada N, Futagami $\mathrm{S}$, et al. Myopic choroidal neovascularization: a 10-year follow up. Ophthalmology. 2003;110:1297-305.

31. Gomi F, Nishida K, Oshima Y, Sakaguchi H, Sawa M, Tsujikawa M, et al. Intravitreal bevacizumab for idiopathic choroidal neovascularization after previous injection with posterior subtenon triamcinolone. Am J Ophthalmol. 2007;143:507-9.

32. Carneiro AM, Mendonça LS, Falcão MS, Fonseca SL, Brandão EM, Falcão-Reis FM. Comparative study of $1+P R N$ ranibizumab versus bevacizumab in the clinical setting. Clin Ophthalmol. 2012;6:1149-57.

33. Ferrara N, Damico L, Shams N, Lowman H, Kim R. Development of ranibizumab, an anti-vascular endothelial growth factor antigen binding 
fragment, as therapy for neovascular age-related macular degeneration. Retina. 2006;26:859-70.

34. Yamamoto I, Rogers AH, Reichel E, Yates PA, Duker JS. Intravitreal bevacizumab (Avastin) as treatment for subfoveal choroidal neovascularisation secondary to pathological myopia. Br J Ophtalmol. 2007;91:157-60.

35. Chan WM, Lai TY, Liu DT, Lam DS. Intravitreal bevacizumab (avastin) for myopic choroidal neovascularisation: Six-month results of a prospective pilot study. Ophthalmology. 2007;114:2190-6.

36. Gharbiya M, Allievi F, L Mazzeo, Gabrieli CB. Intravitreal bevacizumab treatment for choroidal neovascularization in pathologic myopia:12month results. Am J Ophthalmol. 2009;147:84-93.

37. Konstantinidis L, Mantel I, Zografos L, Ambresin A. Intravitreal ranibizumab as primary treatment for neovascular membrane associated with idiopathic juxtafoveal retina. Graefes Arch Clin Exp Ophthalmol. 2009;247:1567-9.

38. Silva RM, Ruiz-Moreno JM, Rosa P. Intravitreal ranibizumab for myopic choroidal neovascularization:12-month results. Retina. 2010;30:407-12.

39. Monés JM, Amselem L, Serrano A, Garcia M, Hijano M. Intravitreal ranibizumab for choroidal neovascularization secondary to pathologic myopia:12-month results. Eye. 2009;23:1275-80.

40. Lai TY, Chan WM, Liu DT, Lam DS. Intravitreal ranibizumab for the primary treatment of choroidal neovascularization secondary to pathologic myopia. Retina. 2009;29:750-6.

41. Lalloum F, Souied EH, Bastuji-Garin S. Intravitreal ranibizumab for choroidal neovascularization complicating pathologic myopia. Retina. 2010;30:399-406

42. Ikuno $\mathrm{Y}$, Sayanagi K, Soga K. Intravitreal bevacizumab for choroidal neovascularization attributable to pathological myopia:one-year results. Am J Ophthalmol. 2009;147:94-100.

43. Hayashi K, Ohno-Matsui K, Yoshida T. Characteristics of patients with a favorable natural course of myopic choroidal neovascularization. Graefes Arch Clin Exp. Ophthalmol. 2005;243:13-9.
44. Arias L, Planas N, Prades S, Caminal JM, Rubio M, Pujol O, et al Intravitreal bevacizumab (Avastin) for choroidal neovascularisation secondary to pathological myopia:6-month results. $\mathrm{Br} \mathrm{J}$ Ophthalmol. 2008;92:1035-9.

45. Sayanagi K, Ikuno Y, Soga K, Wakabayashi T, Tano Y. Marginal crack after intravitreal bevacizumab for myopic choroidal neovascularization. Acta Ophthalmol. 2008;85:50-4

46. Mordenti J, Cuthbertson RA, Ferrara N, Thomsen K, Berleau L, Licko $\mathrm{V}$, et al. Comparisons of the intraocular tissue distribution, pharmacokinetics, and safety of 125l-labeled full-length and Fab antibodies in rhesus monkeys following intravitreal administration. Toxicol Pathol. 1999;27:536-44.

47. Chan WM, Lai TY, Liu DT, Lam DS. Intravitreal bevacizumab (Avastin) for myopic choroidal neovascularisation: 1-year results of a prospective pilot study. Br J Ophthalmol. 2009;93:150-4.

48. Gharbiya M, Allievi F, Mazzeo L, Gabrieli CB. Intravitreal bevacizumab treatment for choroidal neovascularization in pathologic myopia: 12-month results. Am J Ophthalmol. 2009;147:84 -93.

49. Kelly SP, Barua A. A review of safety incidents in England and Wales for vascular endotelial growth factor inhibitor medications. Eye. 2011;25:710-6.

50. Shah CP, Garg SJ, Vander JF, Brown GC, Kaiser RS, Haller JA, et al Outcomes and risk factors associated with endophthalmitis after intravitreal injection of anti-vascular endothelial growth factor agents. Ophthalmology. 2011;118:2028-34.

51. Bakri SJ, Larson TA, Edwards AO. Intraocular inflammation following intravitreal injection of bevacizumab. Graefes Arch Clin Exp Ophthalmol. 2008;246:779-81.

52. Johnson D, Hollands $H$, Hollands $S$, Sharma S. Incidence and characteristics of acute intraocular inflammation after intravitreal injection of bevacizumab: a retrospective cohort study. Can J Ophthalmol. 2010;45:239-42. 


\section{Avaliação dos Resultados do Tratamento Antiangiogénico na Neovascularização Coroideia Associada à Miopia Patológica Acta Med Port 2014:27:49-58}

Publicado pela Acta Médica Portuguesa, a Revista Científica da Ordem dos Médicos

Av. Almirante Gago Coutinho, 151

1749-084 Lisboa, Portugal.

Tel: +351 218428215

E-mail: submissao@actamedicaportuguesa.com

www.actamedicaportuguesa.com

ISSN:0870-399X | e-ISSN: 1646-0758

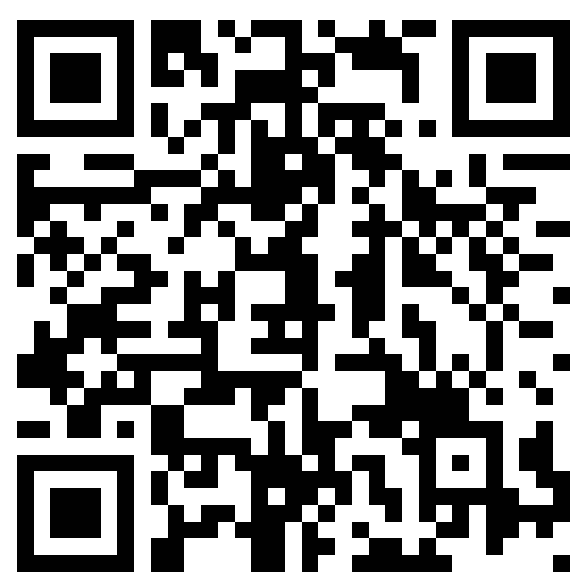

